\title{
Epipage 2 : une étude épidémiologique nationale pour mieux connaître la prise en charge et le devenir des enfants grands prématurés
}

\author{
Epipage 2: a national epidemiological study for enhanced knowledge \\ of care and subsequent development of very premature children
}

\author{
P.-Y. Ancel (pour le groupe Epipage 2)
}

(C) Springer-Verlag France 2010

Chaque année en France, plus de 10000 enfants naissent grands prématurés $(<33$ semaines d'aménorrhée [SA]). Les progrès réalisés en 20 ans ont permis d'améliorer la survie d'enfants de plus en plus immatures. Ces enfants sont désormais plus nombreux à la naissance et plus nombreux à survivre, ce qui rend indispensable le recueil d'informations au-delà des premières semaines de vie. Mais, évaluer leur devenir n'est pas simple et requiert la mise en place d'enquêtes épidémiologiques de grande taille, sur une base populationnelle, avec un suivi à long terme; des conditions qui garantissent la généralisation des résultats tout en rendant compte de la diversité des pratiques et des modes d'organisation des soins. C'est aussi pour ces raisons qu'elles restent peu nombreuses. En Europe, seules trois cohortes ont été mises en place : Epicure en Angleterre et en Irlande, Epipage en France et Epibel en Belgique.

L'étude Epipage, menée sur des enfants grands prématurés nés en 1997, a montré qu'en dépit des progrès accomplis, la mortalité et la morbidité néonatales restaient élevées et les handicaps fréquents. Elle a aussi permis de rendre compte d'une réalité complexe. Si près de $40 \%$ des grands prématurés présentaient une séquelle motrice ou sensorielle ou un retard intellectuel à cinq ans (quatre fois plus qu'un enfant né à terme), les formes les plus graves ne concernaient que $5 \%$ des enfants. Cependant, certains facteurs comme le retard de croissance, la situation sociale familiale et surtout l'âge gestationnel influençaient fortement le risque de séquelles, illustrant la grande variabilité du pronostic en fonction de

P.-Y. Ancel $(\bowtie)$

Inserm unité 953, unité de recherche épidémiologique en santé périnatale et santé des femmes et des enfants, hôpital Tenon, F-75020 Paris, France

e-mail : yves.ancel@tnn.aphp.fr

UMR S953, UPMC, université Paris-VI, F-75005 Paris, France

Groupe Epipage 2, équipes cliniques et de recherche des régions françaises participantes ces situations. Enfin, Epipage a souligné les problèmes posés par la prise en charge à la naissance, particulièrement aux limites de la viabilité, mais plus encore les besoins de suivi et d'évaluation des interventions proposées pendant l'enfance. Comme d'autres études de cohorte en population, elle a dû faire face au problème des perdus de vue, un quart des enfants n'ayant pu être évalués à cinq ans. La non-prise en compte de ces enfants tend à sous-estimer les difficultés, déficiences et handicaps. La mise en place des réseaux périnatals et surtout des réseaux de suivi devrait permettre d'améliorer ce point à l'avenir.

Depuis la fin des années 1990, des changements ont été observés. En France, les données les plus récentes montrent que les grands prématurés sont plus nombreux qu'en $1997(+12 \%)$ et plus nombreux à survivre $(+20 \%)$. Le regard sur la grande prématurité a aussi changé, les interrogations portant maintenant essentiellement sur le devenir cognitif et comportemental des enfants. Concernant l'organisation des soins, le plan périnatalité s'est concrétisé par la mise en place des réseaux de santé en périnatalité, mais on dispose de peu de données sur l'évaluation de ces réseaux. Les changements ont également concerné la prise en charge néonatale avec un recours à de nouveaux traitements et protocoles, nutritionnels notamment, rendant nécessaire l'actualisation des données et la mesure de leurs effets sur le développement ultérieur de l'enfant. La question de la prise en charge des enfants à l'issue de l'hospitalisation se pose, car il s'agit d'un domaine où le niveau de preuve sur l'efficacité des interventions est faible. Enfin, plusieurs domaines touchant aux causes et aux conséquences de la grande prématurité restent encore peu connus et explorés (aspects génétiques, rôle de marqueurs biologiques, nouvelles techniques d'imagerie cérébrale). C'est dans ce contexte qu'une nouvelle étude nationale, l'étude Epipage 2, doit débuter en 2011 en France. Elle a pour objectifs :

- de mieux connaître le devenir des enfants grands prématurés ; 
- d'évaluer les effets de l'organisation des soins et des pratiques médicales sur la santé et le développement des enfants prématurés ;

- d'améliorer les connaissances sur les causes et les conséquences de la prématurité :

- en recherchant des marqueurs biologiques des complications en relation avec la prématurité ;

- en étudiant la valeur pronostique des lésions cérébrales sur le développement, à partir de l'échographie transfontanellaire, de l'imagerie par résonance magnétique (IRM) conventionnelle et des séquences particulières en IRM (tenseur de diffusion, tractographie, spectroscopie);

- de mieux connaître les parcours des familles et les processus de décision au moment de la naissance et en soins intensifs ;

- d'estimer et de prévoir les besoins de prise en charge médicale et éducative.

Pour atteindre ces objectifs, Epipage 2 sera menée sur la population des enfants grands prématurés $(<33 \mathrm{SA})$ et prématurés modérés (33-34 SA) nés dans les 22 régions de France métropolitaine et en Martinique. Les autres régions d'outre-mer, en particulier la Guadeloupe et la Réunion, pourraient prochainement rejoindre le projet. L'étude, qui débutera en avril 2011, permettra de suivre plus de 6000 enfants prématurés jusqu'à l'âge de 12 ans. Un groupe témoin de 1000 enfants nés à 39-40 SA sera constitué et servira de référence pour les indicateurs de santé et de développement.

Constituer une cohorte représente une méthodologie de choix pour apporter des réponses sur le pronostic, l'étiologie et l'évaluation des pratiques médicales pour l'étude de populations à haut risque et l'identification de stratégies de prévention et de prise en charge. Mais, l'originalité d'Epipage 2 réside dans la couverture nationale de cette population d'enfants et des équipes qui en ont la charge, l'exploration de dimensions de la santé et du développement peu couvertes jusqu'ici, et la mise en œuvre de projets de recherche complémentaires venant se greffer sur l'étude pour répondre à des questions dans le champ de l'imagerie cérébrale, des biomarqueurs et de l'éthique. Pour mener à bien ce projet, Epipage 2 s'appuie sur ses partenaires « périnatals » que sont les sociétés savantes, Société française de médecine périnatale, Société française de néonatologie, Groupe d'étude en néonatologie d'Île-de-France et Société francophone d'imagerie pédiatrique et prénatale, et sur la fondation PremUp, une fondation de coopération scientifique sur la grossesse et la prématurité. Elle bénéficie d'un financement pour les trois premières années dans le cadre du programme « Très grandes infrastructures de recherche » de l'institut de recherche en santé publique. Mais, l'implication des équipes obstétrico-pédiatriques reste la clé de la réussite du projet, car elles sont en première ligne pour informer les familles, assurer le recrutement des enfants et garantir un recueil rigoureux des données en maternité et en service de néonatologie. De la solidité de ce socle dépendra la qualité des projets et des investigations ultérieures. C'est en intégrant les équipes dans la formulation des questions posées par la prise en charge de ces enfants qu'Epipage 2 sera en mesure de répondre au mieux aux problématiques qui se posent en France actuellement.

\section{Groupe Epipage 2}

Alsace : J. Matis, P. Khun, D. Astruc, B. Langer (Strasbourg) ; Aquitaine : L. Joly-Pedespan (Bordeaux), P. Chabanier (Bordeaux), X. Hernandorena (Bayonne) ; Auvergne : B. Lecomte, M. Accoceberry, D. Lemery (Clermont-Ferrand) ; Basse-Normandie : G. Beucher, M. Dreyfus, B. Guillois (Caen) ; Bourgogne : P. Sagot, S. Couvreur, A. Burguet, J.-B. Gouyon (Dijon) ; Bretagne : D. Soupre (Vannes), J. Sizun (Brest), P. Pladys (Rennes), R.-P. Dupuy (Saint-Brieuc), F. Rouget (Rennes), A. Beuchée (Rennes); Centre : A. Favreau, E. Saliba (Tours); ChampagneArdenne : P. Morville (Reims) ; Franche-Comté : G. Thiriez (Besançon) ; Haute-Normandie : S. Marret, L. Marpeau (CHU de Rouen); Île-de-France : P. Boileau (Clamart), D. Mitanchez (Paris), M. Granier (Évry), X. Durrmeyer (Créteil), B. Carbonne (Paris), G. Kayem (Créteil) ; LanguedocRoussillon : P. Boulot, G. Cambonie, B. Ledésert, G. Criballet, H. Daudé, O. Plan, M. Bienfait (Montpellier) ; Limousin : S. Ketterer, F. Mons, A. Bédu (Limoges) ; Lorraine : J. Fresson, J.-M. Hascoët, O. Thiebaugeorges, M.-H. Binet, A. Milton, R. Vieux, P. Judin (Nancy) ; Midi-Pyrénées : C. Arnaud (Inserm U558, Toulouse), C. Alberge, C. Casper, C. Vayssière (Toulouse) ; Nord-Pas-de-Calais : V. Pierrat, D. Subtil, P. Truffert (Inserm U953, Lille) ; PACA et Corse : U. Simeoni, C. D'Ercole, C. Gire (Marseille), A. Bougain (Nice) ; Pays de la Loire : J.-C. Rozé, N. Winer, B. Branger (Nantes) ; Picardie : J. Gondry, G. Krim (Amiens) ; Poitou-Charentes : K. Husseini, F. Pierre, M.-F. Bouthet (Poitiers) ; Rhône-Alpes : O. Claris, J.-C. Picaud (Lyon), T. Debillon et C. Cans (Grenoble), M. Debeir (Chambéry), H. Patural (Saint-Étienne), S. Rubio-Gurung ; Martinique : S. Châlons (Fort de France) ; Unité 953 : F. Goffinet, M. Kaminski, L. Foix-L'Hélias, B. Khoshnood, G. Kayem, C. Lebeaux, M. Durox, B. Blondel, M.-J. Saurel-Cubizolles, L. Marchard-Martin, P.-Y. Ancel ; Radiologues : C. Adamsbaum (Paris), J.-F. Chateil (Bordeaux), K. Chaumoître (Marseille), C. Sembely-Taveau (Tours), C. Treguier (Rennes), N. Méjean (Dijon), M. Brasseur Daudruy (Rouen), E. Schmitt (Nancy), B. Richter (Caen). Régions qui pourraient rejoindre le projet : Guadeloupe et Réunion. 